\title{
Wind Tunnel Tests of Evolved Mars Tumbleweed Concepts
}

\author{
Jeffrey Antol, ${ }^{*}$ Steven B. Harris, ${ }^{\dagger}$ and Gregory A. Hajos ${ }^{\star}$ \\ NASA Langley Research Center, Hampton, VA 23681 \\ Christopher V. Strickland ${ }^{\S}$ \\ NASA Wallops Flight Facility, Wallops Island, VA 23337
}

\begin{abstract}
The Mars Tumbleweed rover is a concept for an exploration vehicle that would derive mobility through use of the winds on Mars. The Tumbleweed would conduct random sampling of the surface and atmosphere, traveling long distances over broad regions of the Martian surface. NASA Langley Research Center (LaRC) has developed notional concepts of Tumbleweed rovers and has been studying the aerodynamics of these concepts through wind tunnel testing and computational fluid dynamics analysis. This paper will provide an overview of the latest wind tunnel tests, which were conducted in the fall of 2004 in the LaRC Basic Aerodynamic Research Tunnel (BART). Various configurations of three Tumbleweed concepts, evolved from similar concepts tested in the BART during the previous year, were tested to determine and assess their drag characteristics. Descriptions of the models are provided as well as an overview of the BART facility and the strain gage balance used in the test. A description of the test methodology and the resulting data is presented followed by conclusions drawn from the results.
\end{abstract}

\section{Nomenclature}

$\begin{array}{ll}\alpha & =\text { angle of attack } \\ A O A & =\text { angle of attack } \\ B A R T & =\text { Basic Aerodynamic Research Tunnel } \\ C d & =\text { coefficient of drag } \\ C F D & =\text { computational fluid dynamics } \\ f t & =\text { feet } \\ f t / s e c & =\text { feet per second } \\ J P L & =\text { Jet Propulsion Laboratory } \\ \mathrm{LaRC} & =\text { Langley Research Center } \\ \mathrm{lb} & =\text { pound } \\ \mathrm{m} & =\text { meter } \\ \mathrm{mm} & =\text { millimeter } \\ \mathrm{m} / \mathrm{s} & =\text { meters per second } \\ \mathrm{mV} & =\text { millivolt } \\ N & =\text { Newton } \\ R e & =\text { Reynolds number } \\ S L & =\text { stereolithography } \\ \mathrm{sec} & =\text { second } \\ V & =\text { Fluid Speed }\end{array}$

\footnotetext{
* Aerospace Engineer, Architectures, Missions \& Science Branch, MS 462, Non-member AIAA

${ }^{\dagger}$ Aerospace Engineer, Exploration System Engineering Branch, MS 489, Non-member AIAA

* Aerospace Engineer, Architectures, Missions \& Science Branch, MS 462, AIAA member

$\S$ Aerospace Engineer, Mechanical Systems Branch, MS 548, AIAA member
} 


\section{Introduction}

$\mathrm{T}$ he wind on the surface of Mars has been studied extensively since the first in-situ measurements were made by the Viking landers in the 1970s. The idea for using the wind to propel a rover was first developed shortly thereafter by Jacques Blamont of the National Center for Space Studies in France. The idea evolved into the University of Arizona Mars Ball, an inflatable rover which also used sequenced inflation and deflation of air bags to aid mobility. ${ }^{1}$ A purely wind-driven rover did not appear feasible at the time and detailed research into the concept was discontinued. In the early 2000s, teams at the NASA Langley Research Center (LaRC) ${ }^{2}$ and the Jet Propulsion Laboratory (JPL) ${ }^{3}$ developed concepts for a wind-driven rover called the "Tumbleweed." The JPL effort has focused on inflatable concepts based on airbag landing technology, while LaRC has focused on concepts based on lightweight deployable structures (figure 1). The purpose of the effort is to develop a rover that could travel greater distances than conventional wheeled rovers, currently capable of traveling only several kilometers, explore rough terrain such as that found in canyons and gullies, and be inexpensive to enable deployment of multiple vehicles on a single mission. Because the Tumbleweeds are randomly propelled by the winds, they are ideal for conducting general surveys of broad regions on the Martian surface, serving as scouting missions for more advanced rovers, landers and human explorers. However, utilizing the wind for mobility on Mars is a challenging task. The Martian winds average approximately 7 meters per second $(\mathrm{m} / \mathrm{s})$, with periods of storms gusting to $10-15 \mathrm{~m} / \mathrm{s}$, while dust storms can reach $25-35 \mathrm{~m} / \mathrm{s}$. But the density is about 80 times less when compared to Earth and the static pressure on the surface of Mars is about 1 percent that of Earth at sea level. In order for a Tumbleweed rover to achieve mobility in this environment, it would need to be 4-6 meters in diameter when deployed and have a total mass no greater than 20 kilograms, which includes the structure, power, and communication systems as well as an optimized set of miniature science instrumentation.
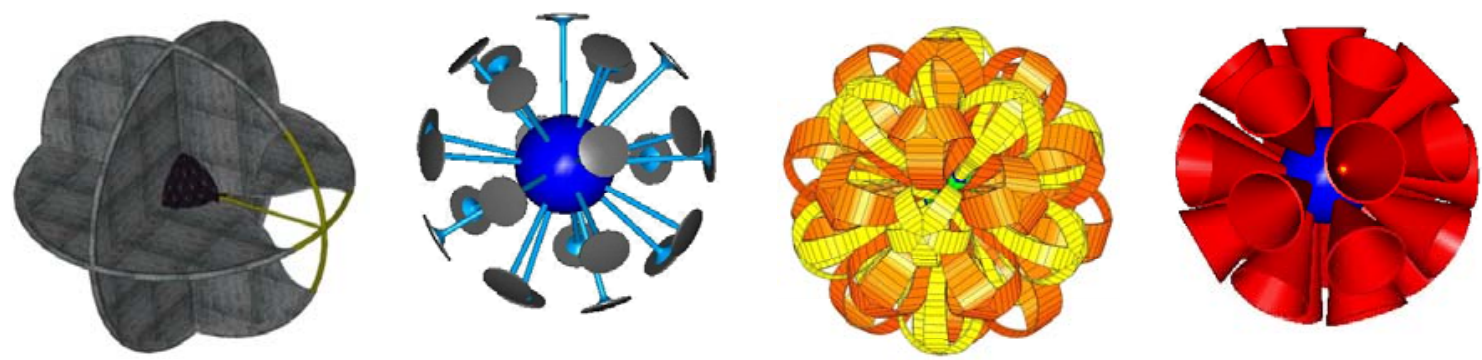

Figure 1. NASA LaRC Tumbleweed Concepts, from left to right: Box-Kite, Dandelion, Eggbeater Dandelion, and Tumble-Cup

The goal of the LaRC team, using deployable open structure concepts, is to achieve drag coefficients $(\mathrm{Cd})$ greater than that of a simple sphere (0.5) in order to minimize the size of the Tumbleweed and/or increase the instrument mass capability. Thus, LaRC has initiated a threefold, evolutionary test strategy to measure the drag characteristics and better understand the Tumbleweed concepts: (1) in the free stream, at the appropriate Reynolds number (Re); (2) on the surface, in a simulated Mars boundary layer at the appropriate Reynolds number; and (3) in a Mars relevant environment, at the appropriate temperature, pressure and density. The tests discussed in this paper cover the first category, free stream testing. Using the results from an initial set of tests conducted in 2003 employing the LaRC Basic Aerodynamics Research Tunnel (BART), ${ }^{4}$ several evolved Tumbleweed designs were created and a set of follow-on tests was planned and conducted in the BART in 2004. The associated wind tunnel models, test configurations, and the balance data are presented in the following sections.

\section{Model Design}

In order to match the expected Reynolds numbers for the surface of Mars $(\operatorname{Re}=50,000-125,000)$ from average winds to storm gusts, small-scale models were needed as well as very low wind tunnel test speeds to meet the expected Reynolds numbers. Models on the order of 1 foot in diameter were selected to ease manufacturing, to simplify model changes, and to match the test section criteria of the wind tunnel. Three evolved Tumbleweed designs were selected for the 2004 tests (figure 2). The first Dandelion concept employed a curved beam structure, named the Eggbeater Dandelion. The Box kite model used cloth sails as opposed to the previous test, which used solid "sail" panels. The other used deeply cupped pads (Cup-Pad Dandelion) as opposed to the slightly cupped pads 
of the original Dandelion model from the 2003 tests. The Eggbeater Dandelion and the Cloth Sail Box Kite models were new developments, while the Cup Pad Dandelion used many components from the original Dandelion model.
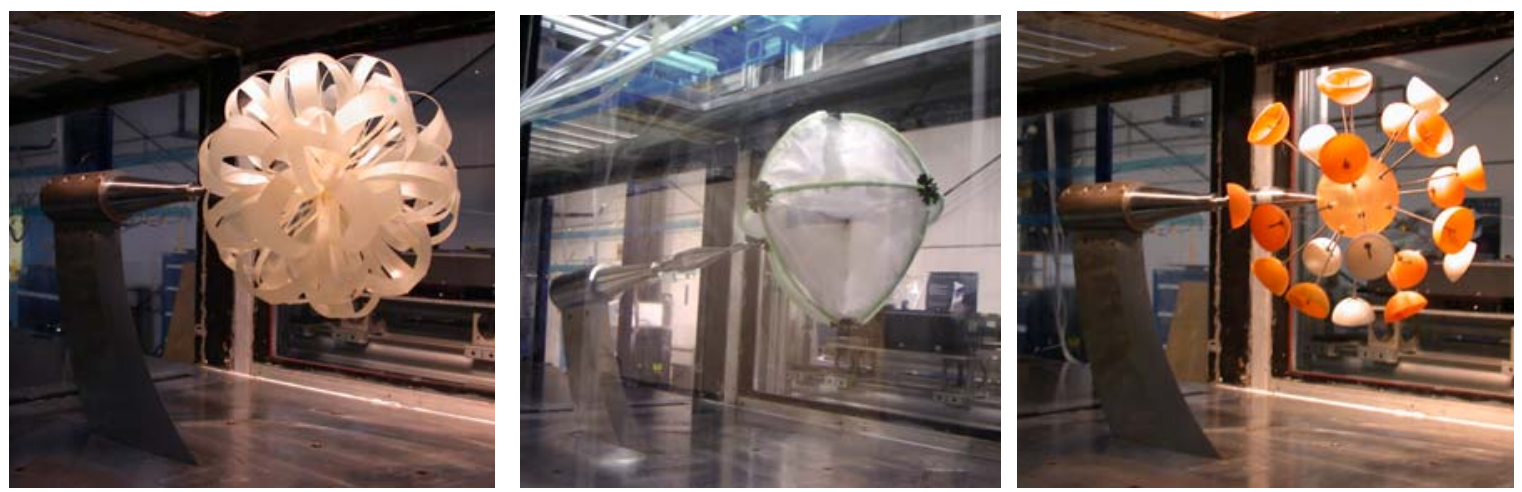

Figure 2. Evolved LaRC Tumbleweed concepts in BART test section

(from left to right, Eggbeater Dandelion, Cloth Sail Box Kite, and Cup-Pad Dandelion)

\section{A. Eggbeater Dandelion Model}

The Eggbeater-Dandelion Tumbleweed was derived from the original Dandelion Tumbleweed concept (depicted in figure 1) - a central core with radial spoke legs that "provide load bearing mechanical isolation for the core and mechanical support for drag producing surfaces." A non-linear impact dynamics analysis of the original Dandelion demonstrated that radial legs would encounter structural buckling problems at the required impact velocities. ${ }^{6}$ The straight spoke legs of the Dandelion were thus replaced with legs of a curved beam design, with each leg resembling an eggbeater or whisk, hence the name "Eggbeater Dandelion" (figure 3). The width of each ribbon on each leg is tapered radially from the core attachment point to provide additional drag producing surface area. Three and five ribbon legs were combined to allow interlocking of the ribbons of adjacent Eggbeater legs to enable more drag surface area without leg-to-leg physical interference. To maintain spherical symmetry, the configuration included thirty-two legs with attachment points coincident with the vertices of the two largest Platonic solids, the Dodecahedron (20 vertices) and the Icosahedron (12 vertices).
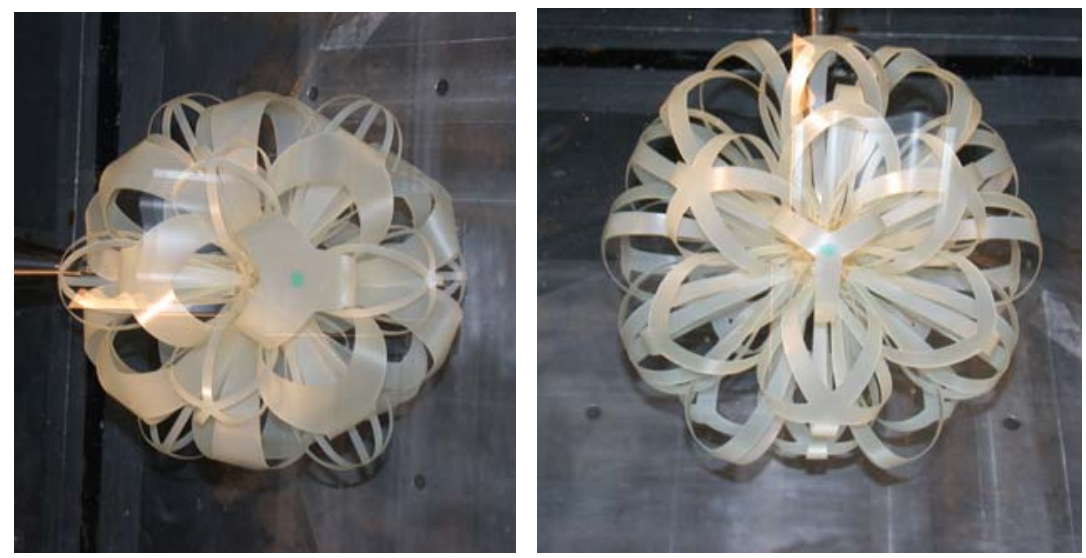

Figure 3. Eggbeater Dandelion models

The core design chosen to accomplish the compounded Dodecahedron/Icosahedron vertice attach points for the wind tunnel model was a Truncated Icosahedron. A Truncated Icosahedron is essentially a soccer ball with planar patches. The centers of the 20 hexagon patches of a soccer ball correspond with the Dodecahedron vertices and the centers of the 12 pentagon patches of a soccer ball correspond with the Icosahedron vertices. Figure 4 depicts the general core scheme of the Eggbeater-Dandelion Tumbleweed wind tunnel model with one three ribbon leg and one five ribbon leg installed. Each of the 12 pentagon patches has five 0.25 inch wide by 0.026 inch thick by 0.45 inch deep (in the radial direction) slots to accept the five tabs of the five ribbon legs (see figure 5). Similarly, the 20 hexagonal patches of the core have three slots each to accept the three tabs of the three ribbon legs. A 1/4-20 inch screw thread insert was installed in the core to provide for attachment to the wind tunnel sting assembly. The cores 
for the wind tunnel models were manufactured from SI-10 material by the NASA LaRC model shop using the Viper $^{\text {TM }}$ stereolithography (SL) system from 3-D Systems.

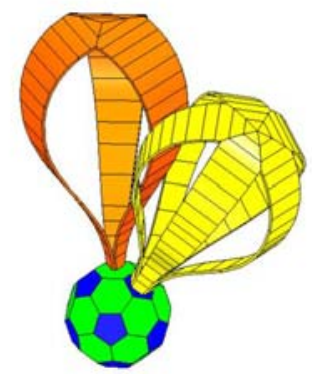

\section{Figure 4. Eggbeater Dandelion truncated icosahedron core with a three and five ribbon leg installed}

The legs were cut from 0.02-inch thick woven fiberglass reinforced epoxy sheet by Custom Cutting Technologies, Inc. as per figure 5. The three ribbon and five ribbon legs were cut at three different taper angles. The smallest taper angle for each was called "Low," the middle angle was called "Medium," and the largest taper angle was called "High." The surface areas of three ribbon "Low" and five ribbon "Low" are approximately equal. Similarly, the three and five ribbon "Medium" areas are approximately equal as are both "Highs." This was done to create a through flow path with no "easy" route. It was hypothesized that flow tripping and reformation would lead to drag coefficients greater than that of a solid sphere. The primary test configurations combined the "Low-toLow," "Medium-to-Medium," and "High-to-High" taper angle three and five ribbon legs. Since the tabs of the legs were held in the slots of the core by friction and/or interference fit, secondary testing was done by removing the legs from the primary test configuration and recombining 1) the three ribbon "Lows" with the five ribbon "Mediums," 2) the three ribbon "Mediums" with the five ribbon "Highs," and 3) the three ribbon "Highs" with the five ribbon "Lows."
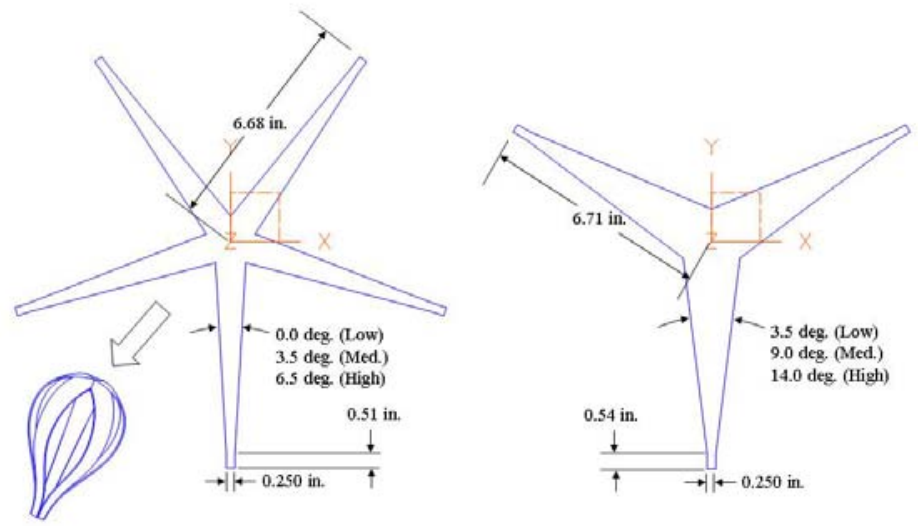

Figure 5. Drawing for manufacture of three and five ribbon Eggbeater Dandelion legs

\section{B. Cloth Sail Box Kite Model}

A commercially available watercraft radar reflector, consisting of three orthogonal circular aluminum plates, was used as the Box Kite model in the 2003 BART wind tunnel tests. A new Box Kite model was constructed for the 2004 tests in order to examine a more realistic configuration with cloth sails, instead of the rigid panels, as would be employed in the Martian Tumbleweed. It was anticipated that an increased drag effect would be observed due to "billowing" effects provided by the cloth sails.

The cloth sail Box Kite model was constructed from common, off-the-shelf hobby materials (figure 6). The outer ring frame structure of the model was composed of three orthogonal hoops of K'nex ${ }^{\mathrm{TM}}$ tubing and associated connectors. The sails were created using $0.75 \mathrm{oz} / \mathrm{yd}$ rip-stop nylon covering each quadrant section of each hoop. A central core was fabricated using a 2-inch wooden sphere and connected radially to the hoop "nodes" using \#4 threaded rods. A 1/4-20 inch screw thread insert was also installed in the wooden sphere to provide for attachment to the wind tunnel sting assembly. The position of the insert was chosen in order to orient one of the sails 
perpendicular to the flow (i.e., flat plate configuration), so as to examine a different mode from the solid model used in the 2003 test, which was oriented to provide flow into one of the interior corners (i.e., where the sails intersect at the core).
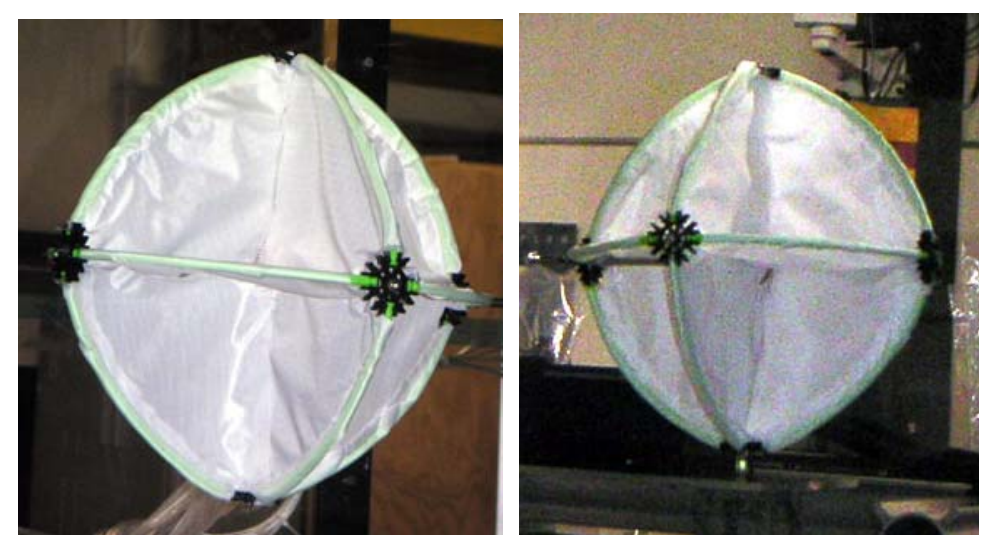

Figure 6. Cloth sail Box Kite model

\section{Wooden Box Kite Model}

A lightweight wooden model was constructed of $1 / 64$ th inch $(0.4$ millimeter $(\mathrm{mm}))$ thick birch plywood to reduce the mass of the Box Kite model from that of the aluminum model used in the previous tests. The three orthogonal 12-inch diameter circular panels were glued and taped into position (figure 7). The wooden Box Kite model used the same sting adaptor as the aluminum model, providing a consistent mounting position and orientation between the two models. It was anticipated that variations in the test results between the aluminum model and the wood model would be a result of the minor difference in diameter and flexibility induced dynamic effects (flutter).

Prior to installation in the wind tunnel, both of the Box Kite models were subjected to winds in excess of $150 \%$ of the planned test speeds to examine the flutter and to verify the structural integrity of the models. The cloth sail and wooden Box Kite models are more flexible than the aluminum model and flutter was experienced during the verification test but no signs of damage were detected.

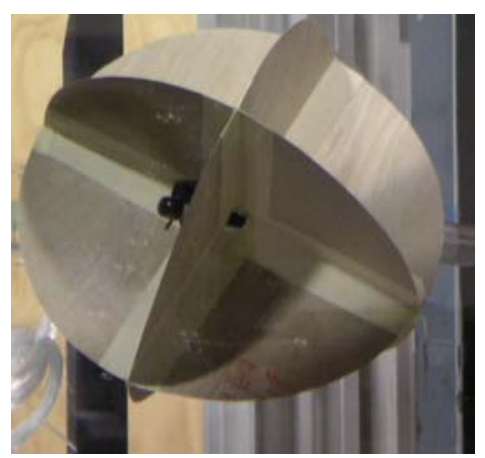

Figure 7. Wooden Box Kite model

\section{Cup-Pad Dandelion Model}

In an effort to optimize the design of the Dandelion Tumbleweed, open hemispherical end pads (cups), known for having a high drag coefficient, were examined. Two variations were constructed; however, the configuration with the open end of the pads facing out from the center of the Tumbleweed was the only one tested due to time constraints (figure 8). The hemispherical cup has a long history of use in anemometers and the aerodynamic characteristics of such a cup are well known in axial flow. One objective of this test was to examine the combined effects of these cups in spherical arrays as presented by the Dandelion Tumbleweed.

The Cup-Pads are constructed of oversized ping pong balls ( $21 / 8$ inch diameter) which were cut in half and mounted on \#4-40 threaded rods of the 24-stem stereolithography hollow sphere core (outer diameter of four inches) used in the 2003 wind tunnel tests. Jam nuts and washers hold the hemispheres in position on the rods. The outside 
diameter of each configuration was kept to 12 -inches to allow comparison with the other 12-inch diameter tumbleweed models.
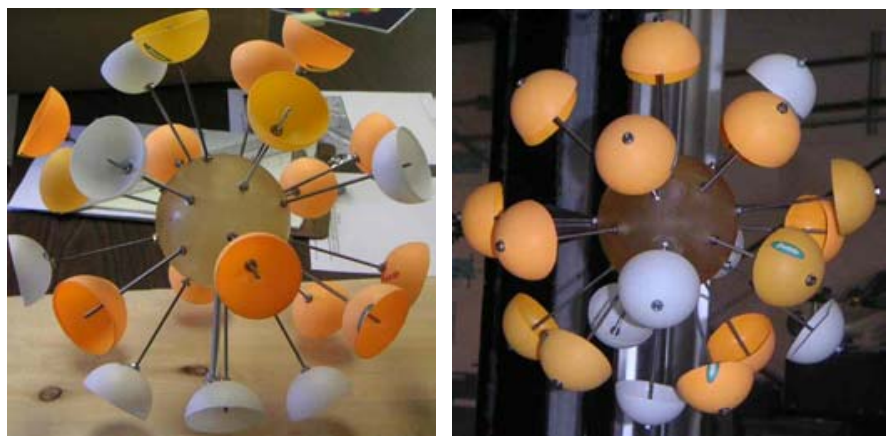

Figure 8. Cup-Pad Dandelion model, outward facing pads (L) and inward facing pads (R)

\section{E. Calibration Spheres}

The 4-inch core of the Dandelion models with the insert holes covered with Kapton tape was used in the previous years test as a calibration sphere to match the test data to theory. Since the strain gauge balance that was used in that test was being recalibrated and therefore not available for our tests, it was included in this set of tests to help correlate the data from each. A 12-inch commercial globe was also used to match the diameter of the models tested. Both spheres will help validate the data from this test and permit direct comparison to the previous test.

\section{Wind Tunnel Facility and Test Configuration}

The Basic Aerodynamic Research Tunnel (BART) was selected due to the low Reynolds number requirements and budget constraints of the project. NASA-Langley's BART tunnel shown in figure 9 is a subsonic, lowturbulence, open-return wind tunnel with a test section 10 feet long and a cross-section dimension of 28 -inch high and 40-inch wide. The test section consists of two 5-foot cells, with large Plexiglas windows that provide access for flow visualization studies. The model angle of attack and data acquisition is remotely controlled using LabView ${ }^{\mathrm{TM}}$ software. The maximum velocity in the test section is 185 feet per second (ft/sec), which yields a Re/ft of 1.4 million. Aerodynamic loads were determined by use of a model balance outfitted with internal strain gauges.

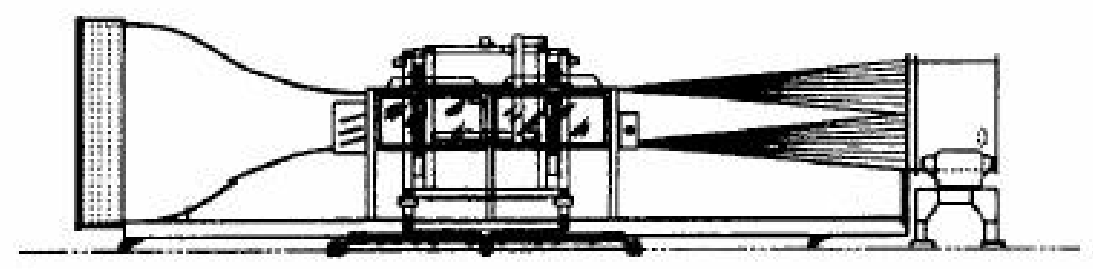

Figure 9. The Basic Aerodynamic Research Tunnel (BART) at NASA Langley Research Center

The model support system (depicted in figure 10) traveled through angles of attack (AOA) from $-5^{\circ}<\alpha<+25^{\circ}$ using a rotating arc sector.

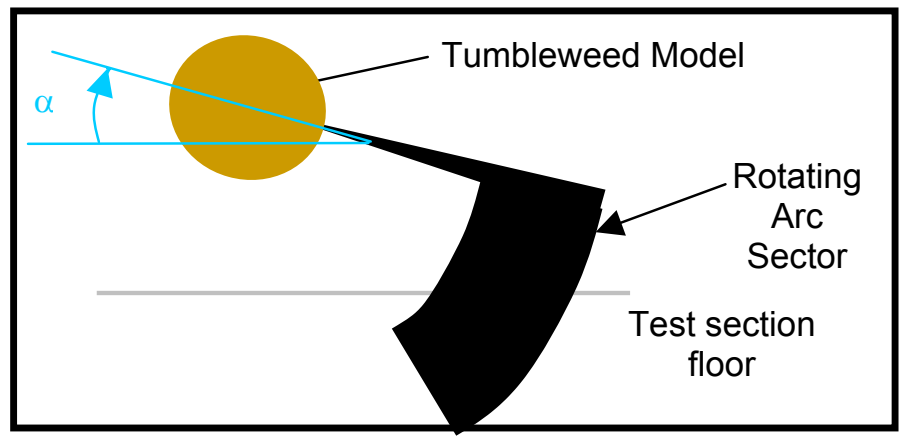

Figure 10. Test configuration - rotating arc sector 
A NASA LaRC balance, Strain Gage Balance 734, was used for the tests. A more sensitive balance was desired but the pitching moment limit was exceeded due to the model weight and the length of the sting attachment. Balance characteristics for the 734 balance are shown in Table 1.

Table 1. NASA LaRC Strain Gage Balance \#734 Characteristics

\begin{tabular}{|c|c|c|c|c|c|c|}
\hline \multirow[t]{2}{*}{ Component } & \multicolumn{2}{|c|}{ Calibration Load range } & \multirow{2}{*}{$\begin{array}{c}\text { Full Scale } \\
\text { Output } \\
(\mathrm{mV} / \mathrm{V})\end{array}$} & \multicolumn{2}{|c|}{ Sensitivity Constant } & \multirow{2}{*}{$\begin{array}{c}\text { Accuracy \% } \\
\text { F. S. } \\
\text { (95\% Conf.) }\end{array}$} \\
\hline & (Lb) or (in-Lb) & $(\mathrm{N})$ or $(\mathrm{Nm})$ & & $\begin{array}{l}(\mathrm{lb} / \mathrm{mV} / \mathrm{V}) \text { or } \\
(\mathrm{in}-\mathrm{lb} / \mathrm{mV} / \mathrm{V})\end{array}$ & $\begin{array}{c}(\mathrm{Nm} / \mathrm{mV} / \mathrm{V}) \text { or } \\
(\mathrm{N} / \mathrm{mV} / \mathrm{V})\end{array}$ & \\
\hline \multirow[t]{2}{*}{ Normal } & 25.0 & 111.206 & 1.189 & 21.0200 & 93.5016 & 0.06 \\
\hline & -25.0 & -111.206 & & & & \\
\hline \multirow[t]{2}{*}{ Axial } & 5.0 & 22.241 & 1.122 & 4.4550 & 19.8168 & 0.12 \\
\hline & 0.0 & 0.000 & & & & \\
\hline \multirow[t]{2}{*}{ Pitch } & 40.0 & 4.519 & 1.264 & 31.6580 & 3.5769 & 0.07 \\
\hline & -40.0 & -4.519 & & & & \\
\hline \multirow[t]{2}{*}{ Roll } & 10.0 & 1.130 & 1.603 & 6.2370 & 0.7047 & 0.16 \\
\hline & -10.0 & -1.130 & & & & \\
\hline \multirow[t]{2}{*}{ Yaw } & 20.0 & 2.260 & 1.303 & 15.3530 & 1.7347 & 0.09 \\
\hline & -20.0 & -2.260 & & & & \\
\hline \multirow[t]{2}{*}{ Side } & 10.0 & 44.482 & 1.317 & 7.5910 & 33.7665 & 0.18 \\
\hline & -10.0 & -44.482 & & & & \\
\hline
\end{tabular}

\section{Wind Tunnel Test}

Smoke visualization tests were performed first to examine the flow as it traveled through and around select models. This was followed by strain gage balance tests, which were conducted at tunnel wind speeds of approximately $20 \mathrm{ft} / \mathrm{sec}$ and $40 \mathrm{ft} / \mathrm{sec}$. The Reynolds number at $20 \mathrm{ft} / \mathrm{sec}(\mathrm{Re}=123,000)$ is within the range of interest; however, at this speed the fan of the BART is near its lowest operating speed and produces somewhat unsteady flow through the test section. Therefore, tests were also conducted at $40 \mathrm{ft} / \mathrm{sec}$, which is at the higher end of the range of interest, in order to achieve a reliable steady flow.

\section{A. Smoke Visualization}

A standard theatrical smoke/fogger machine was positioned on a stand in front of the BART inlet screen, allowing the flow to converge on the front of the model in the first test section. The balance was not installed during the smoke visualization tests due the oil buildup that could occur on the strain gages; so only photographic data was recorded for these tests (still pictures were taken during each test). Wind tunnel speeds for the smoke test were conducted at 11, 20 and $25 \mathrm{ft} / \mathrm{sec}$ and only the Egg Beater Dandelion models were tested in the smoke visualization (figure 11).
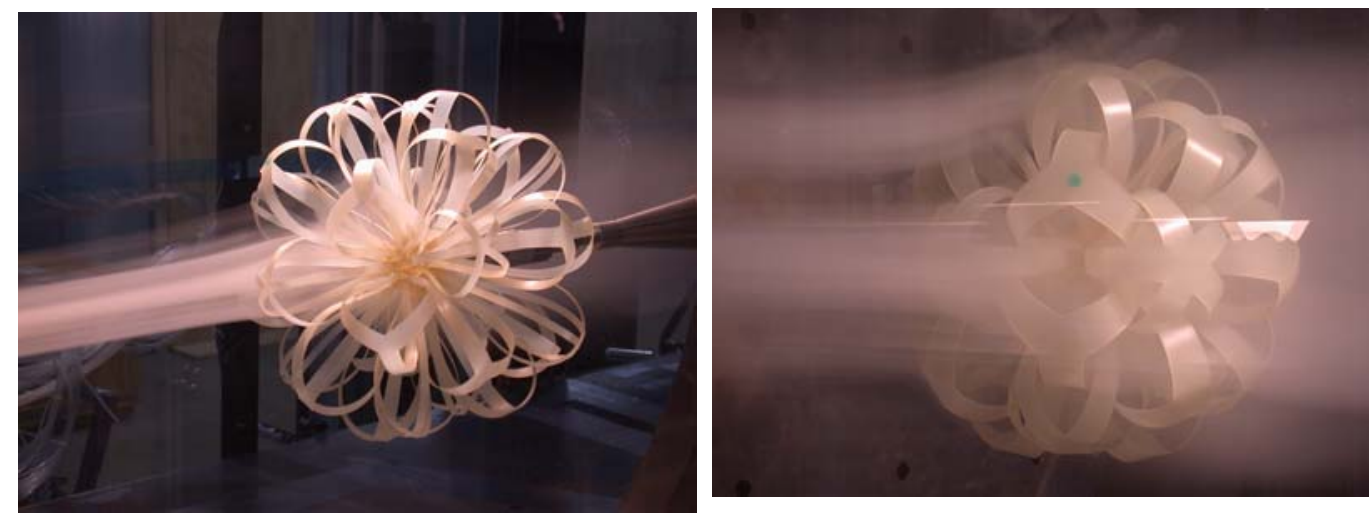

Figure 11. Smoke visualization test of Eggbeater Dandelion configurations 


\section{B. Strain Gage Balance Test}

After each model was mounted to the sting, three tares were performed to determine the loads on the balance due to the model weight at the different angles of attack in order to be subtracted from the test data. Once the tare data was loaded into the LabView ${ }^{\mathrm{TM}}$ software, the tunnel fan was started and the $20 \mathrm{ft} / \mathrm{sec}$ flow tests were initiated. The models were then swept from negative to positive angles of attack while stopping at every degree interval to record data. Every test was completed three times so that an average data set could be generated and to ensure that constant data was being recorded. The tests were then repeated at the $40 \mathrm{ft} / \mathrm{sec}$ wind speed.

\section{Wind Tunnel Data}

After each model was tested at the $20 \mathrm{ft} / \mathrm{sec}$ and $40 \mathrm{ft} / \mathrm{sec}$, the data was compiled, checked for consistency and the averages of all three tests were plotted. All data corresponding to the slower test speed of $20 \mathrm{ft} / \mathrm{sec}$ are plotted using dashed lines while the faster $40 \mathrm{ft} / \mathrm{sec}$ data is plotted using solid lines. The plot of drag coefficient versus the angle of attack for the Dandelion and Box Kite Tumbleweed models and the calibration spheres (4-inch sphere and 12-inch globe) is shown in figure 12. The Dandelion and spheres were tested at $20 \mathrm{ft} / \mathrm{sec}$ and $40 \mathrm{ft} / \mathrm{sec}$ wind speeds, while the Box kite could only be tested at $20 \mathrm{ft} / \mathrm{sec}$ due to the instability of the lightweight models at higher test speeds. The plot of drag coefficient versus the angle of attack for the Eggbeater Dandelion models is shown in figure 13. No negative angles of attack we tested for any of the Eggbeaters because the models were heavier than others and would overload the balance.

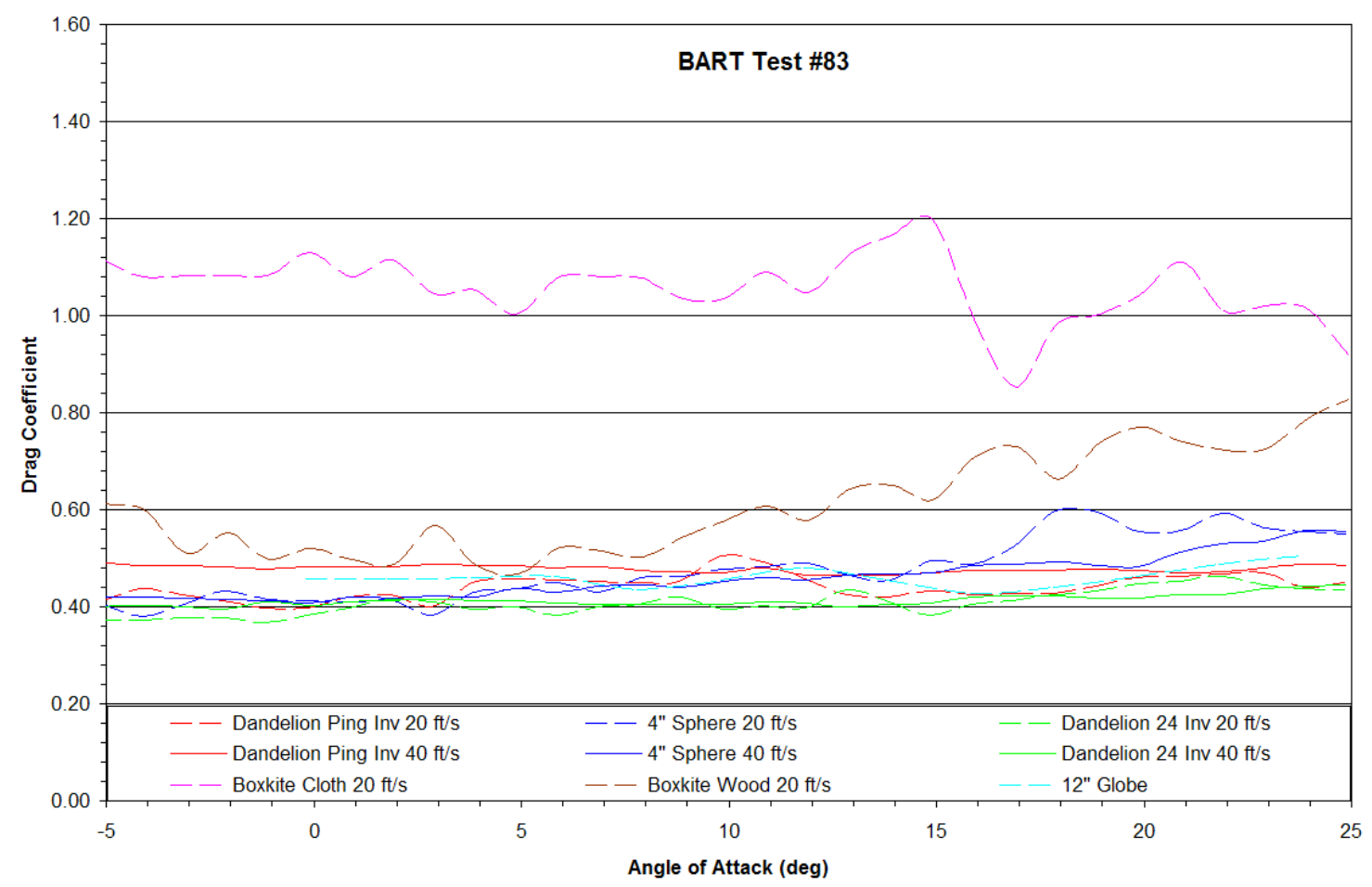

Figure 12. Plot of drag coefficient versus angle of attack for Dandelion, Box Kite and calibration spheres 


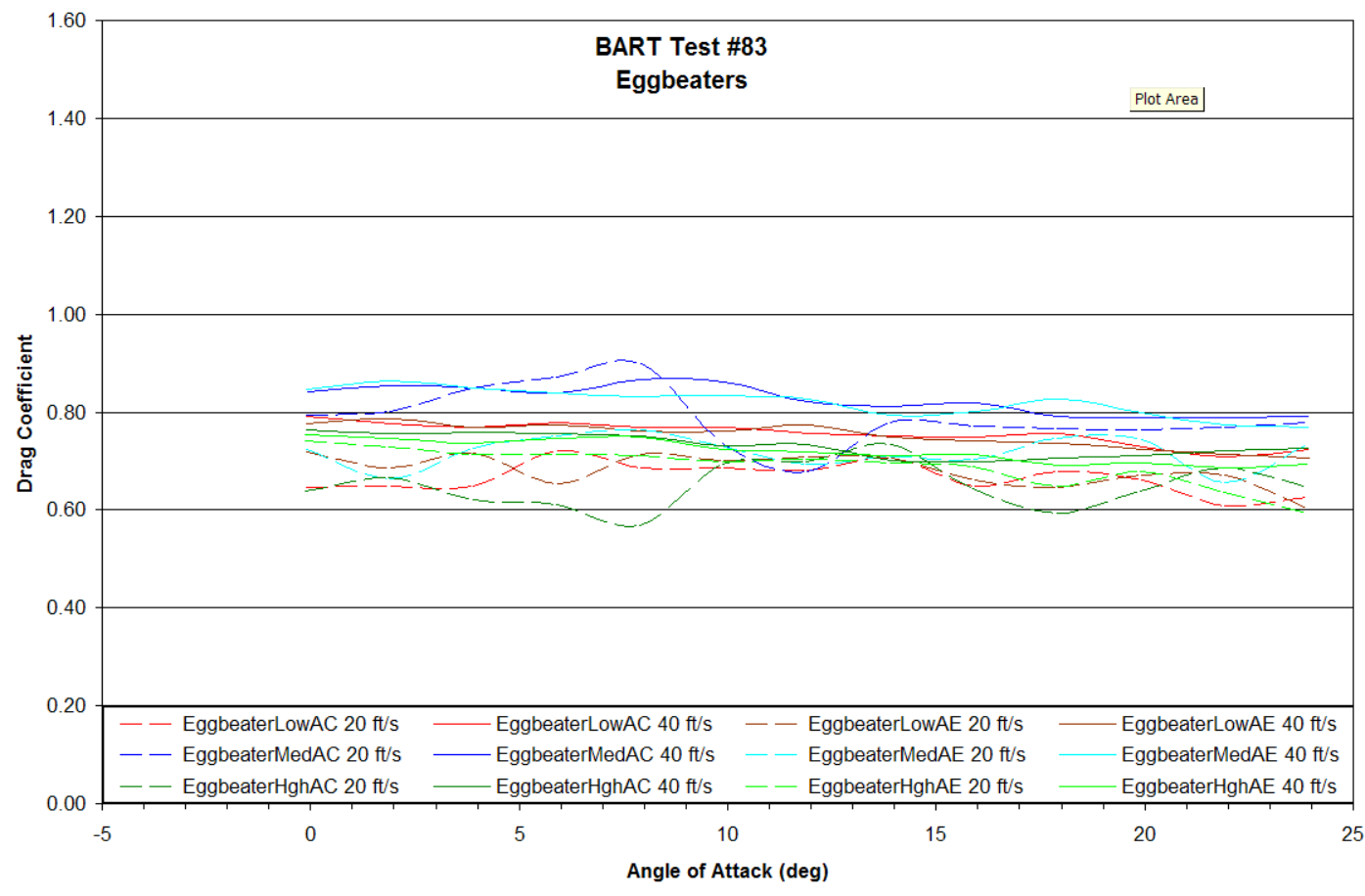

Figure 13. Plot of drag coefficient versus angle of attack for Eggbeater Dandelion configurations

The "AC" and "AE" designation of the eggbeater dandelion Tumbleweeds refers to the "clocking" on the sting (see figure 14). At zero angle of attack, the symmetry axis of the windward five-ribbon leg would be parallel to the oncoming wind tunnel wind direction. For the "AC" tests, the eggbeater dandelion tumbleweed was clocked such that one of the neighboring three ribbon legs was due "north." For the "AE" tests, the eggbeater dandelion tumbleweed was clocked such that one of the neighboring five ribbon legs was due "north."

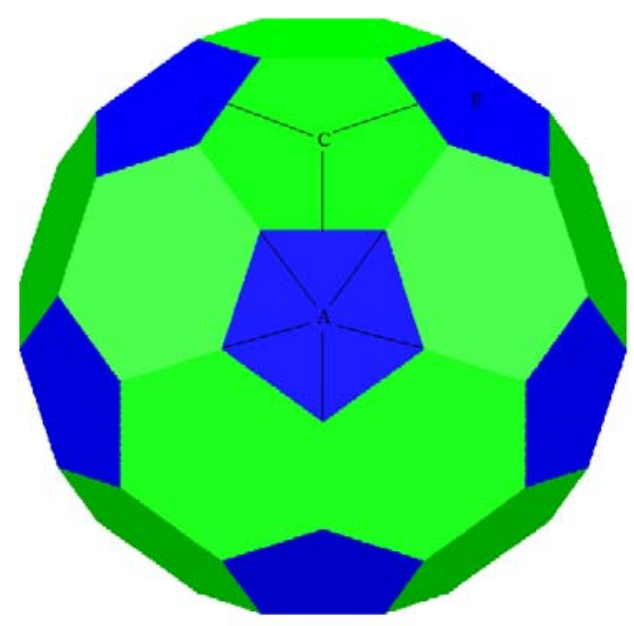

Figure 14. Eggbeater Dandelion naming convention for clocking

\section{Conclusions}

The slower test speed of $20 \mathrm{ft} / \mathrm{sec}$ for each set of tests show widely varying values for drag coefficient as compared with the higher test speed. Since the lower speed is very near the operating limit of the tunnel, it was determined that the flow must be unsteady in the test section and therefore should only be used as a qualitative comparison with the other tests. All quantitative comparisons and conclusions will be based on the higher and steadier $40 \mathrm{ft} / \mathrm{sec}$ test speeds. 
The calculated drag coefficient for both Dandelion models, Inverted Pads and Inverted Ping, shown in figure 12, fall in the same range as the 4-inch and 12-inch calibration spheres. This result indicates that the spoke models may not have a better drag than an equivalent sphere regardless of the drag shapes mounted at the ends. However, this is inconsistent with the previous wind tunnel tests of the baseline Dandelion Tumbleweed ${ }^{4}$ and requires further study and analysis. The Box Kite models show a higher drag coefficient but the models could only be tested at the slower, unsteady, speed of $20 \mathrm{ft} / \mathrm{sec}$. The Box Kite Sail demonstrates a higher drag coefficient than the Box Kite Wood and the rigid Box Kite from the prior test. This was partially due to the different orientation (i.e., flat plate) of the cloth sail Box Kite and the billowing effect of a flexible fabric sail, which aids in adding drag to the Box Kite shape.

All variations of the Eggbeater Dandelion design exhibit drag characteristics that change by no more than 5\% for all models tested. The drag coefficient of the Eggbeaters is higher than a sphere of the same size (Cd of 0.6 to 0.85 for the Eggbeater vs. $\mathrm{Cd} \sim 0.5$ for a sphere). The most likely reason for this, as hypothesized, is that the wind flow is being tripped (disturbed), reformed, and tripped again as the wind flow passes through the open structure of the Eggbeater Dandelion Tumbleweed. Also, as hypothesized, increasing ribbon taper angle (which increases ribbon area) increased the drag coefficient to an inflection point at which the drag coefficient starts to decline. This decline probably occurs when the path of least wind flow resistance is around as opposed to through the Eggbeater Dandelion, thereby approaching sphere-like drag coefficients. It should be noted that even higher drag coefficient numbers are expected if the presently ridged ribbons were replaced with cloth sail ribbons. This effect was observed for the Box Kite due to billowing of the cloth sails.

Data from the 2003 test $^{4}$ as well as the recent 2004 test indicate that the Box Kite has the best overall drag coefficient of the wind tunnel models tested ( $\mathrm{Cd}$ of 0.8 to 1.2). Out of the four Tumbleweed concepts shown in figure 1, the data from these tests indicates that the Box Kite and the Tumblecup show the most promise from a coefficient of drag standpoint $(\mathrm{Cd} \geq 1.0)$. Additional wind tunnel testing is needed on the Eggbeater Dandelion using cloth sail ribbons to examine the effect on the drag coefficient as this may significantly close the drag coefficient gap between the Box Kite / Tumblecup and the Eggbeater Dandelion. Additional tests are also needed on the Box Kite concept at all possible orientations to properly characterize the drag coefficient and correlate with Computational Fluid Dynamics (CFD) models.

\section{Acknowledgments}

The authors would like to thank Dennis Bushnell and the LaRC Creativity \& Innovation (C\&I) initiative and Rich Antcliff and the LaRC Innovation Institute (ii) for their support of Tumbleweed research. The authors would also like to thank Philip Calhoun and Steve Bauer, members of the NASA LaRC Tumbleweed team who assisted with the test, and Luther Jenkins and Richard White, for their operation of the BART facility.

\section{References}

1 Janes, D. M.: "The Mars Ball: A Prototype Martian Rover," AAS 87-272, The Case For Mars III, Part II - Volume 75, AAS Science and Technology Series, Eds. Carol R. Stoker, pp. 569-574, 1989.

2 Antol, J., Calhoun, P., Flick, J., Hajos, G., Kolacinski, R., Minton, D., Owens, R., and Parker J., "Low Cost Mars Surface Exploration: The Mars Tumbleweed,” NASA TM-2003-212411, August 2003.

3 Behar, A., Carsey, F., Matthews, J., Jones, J.: “NASA JPL Tumbleweed Polar Rover,” IEEE Aerospace Conference, Big Sky, Montana, March 2004.

${ }^{4}$ Strickland, C. and Keyes, J.: "Wind Tunnel Tests to Determine Drag Coefficients for the Mars Tumbleweed," AIAA-20050248, 43rd AIAA Aerospace Sciences Meeting and Exhibit, Reno, NV, January 10-13, 2005.

5 Calhoun, P., Harris, S., Raiszadeh, B., Zaleski, K.: "Conceptual Design and Dynamics Testing and Modeling of a Mars Tumbleweed Rover," AIAA-2005-0247, 43rd AIAA Aerospace Sciences Meeting and Exhibit, Reno, NV, January 10-13, 2005.

${ }^{6}$ Jones, J. and White, C.: "Tumbleweed Materials and Structures Study-A Final Report of a JPL Study for Langley Research Center," Jet Propulsion Laboratory, California Institute of Technology, March 18, 2004. 